\title{
Gap junction coding genes and schizophrenia: a genetic association study
}

\author{
Branko Aleksic $\cdot$ Ryoko Ishihara $\cdot$ Nagahide Takahashi \\ Nobuhisa Maeno $\cdot$ Xiaofei Ji $\cdot$ Shinichi Saito \\ Toshiya Inada $\cdot$ Norio Ozaki
}

Received: 4 February 2007 / Accepted: 14 March 2007/Published online: 11 April 2007

(C) The Japan Society of Human Genetics and Springer 2007

\begin{abstract}
The aim of this study was to evaluate the association of genes that encode gap junction forming proteins and schizophrenia. Representative genetic candidates (Panx2 and Cx36) from two families of gap junction genes were selected for analysis. According to the present findings these genes represent both functional and positional candidates for schizophrenia. The sample was comprised of 381 schizophrenic patients, and the same number of matched controls was tested in this study in order to evaluate the possible influence of the aforementioned genes on the pathogenesis of schizophrenia. Four SNPs in the case of Panx2 and two SNPs in the case of Cx36 were selected for analysis. Allele-, genotype- and haplotype-wise association did not yield statistically significant results. These data do not suggest that Panx2 or Cx36 could increase the risk of schizophrenia in the Japanese population.
\end{abstract}

Keywords Gap junction - Panx2 - Cx36 - Schizophrenia Genetic association $\cdot$ Case control $\cdot$ Gamma band oscillation

B. Aleksic $(\bowtie) \cdot$ R. Ishihara $\cdot$ N. Takahashi ·

N. Maeno $\cdot$ X. Ji $\cdot$ S. Saito $\cdot$ N. Ozaki

Department of Psychiatry,

Nagoya University Graduate School of Medicine,

Showa-ku, Tsurumai-Cho, 65,

466-8550 Nagoya, Aichi, Japan

e-mail: branko@med.nagoya-u.ac.jp

T. Inada

Department of Psychiatry, Teikyo University

School of Medicine Chiba Medical Center,

Chiba 299-0111, Japan

\section{Introduction}

Schizophrenia is a severe mental disorder with a global morbid risk of approximately $1 \%$ and a strong genetic component in its etiology. The disease is characterized by abnormal perception, thought disturbances and impaired cognitive function (Sadock and Sadock 2005). One of the present views on the pathogenesis of schizophrenia is related to the disturbed connectivity of neuronal networks (Phillips and Silverstein 2003). The mechanism of the neuronal network depends on two types of synapses, namely, the usual chemical synapses and gap junctions (or electrical synapses) (Connors and Long 2004). A large body of evidence has shown that gap junctions have considerable influence on the stabilization and propagation of synchronized neuronal activity, especially in the beta (12$30 \mathrm{~Hz})$ and gamma $(30-80 \mathrm{~Hz})$ frequency band of EEG (electroencephalogram) (LeBeau et al. 2003; Sohl et al. 2005). The aforementioned had been shown both in in vitro (Whittington et al. 1995; Szabadics et al. 2001; LeBeau et al. 2003; Mann et al. 2005; Traub et al. 2005) and in vivo studies, namely in experiments involving Cx36 (connexin 36) knock out mice (Hormuzdi et al. 2001; Buhl et al. 2003). Furthermore, gap junctions have the capability of bidirectional signal transmission and low-pass filter (LPF) characteristics that are markedly different from properties of chemical synapses. LPF as a term is used for describing the virtue of preferential transmission of sub-threshold potentials and low frequency stimuli that favor synchronous activity of neural networks (an extensive review is available at Sohl et al. 2005).

Two families of gap junction proteins have been described in humans: connexins and pannexins. The connexin family is divided into three subfamilies (alpha, beta and gamma) consisting of more than ten different proteins 
(Rozental et al. 2000); however, Cx36 (subfamily gamma) is preferentially expressed in cell types of neural origin (Condorelli et al. 2000). Moreover, as has been mentioned before, several studies have indicated its role in the promotion of synchrony and power in a gamma frequency range of EEG (Hormuzdi et al. 2001; Buhl et al. 2003). The human pannexin (in a further text referred to as Panx) family consists of Panx1, Panx2 and Panx3. However, Panx 2 is abundantly expressed in the brain and involved in forming gap junctions of principal cells or GABAergic interneurons (gamma amino butyric acid) (Bruzzone et al. 2003).

Regarding schizophrenia, several lines of evidence have showed decreased power and synchrony in the gamma frequency band of EEG during cognitive tasks in comparison to the nonaffected group (Spencer et al. 2003, 2004; Hong et al. 2004; Waberski et al. 2004). In that regard, the reported deficit in gamma band oscillations might be contributed by impaired synchronization of pyramidal cells owing to nonadequate perisomatic inhibition by paralbumin-expressed GABAergic interneurons, (Lewis et al. 2005).

Therefore, regarding gene expression and the reported role in the generation of gamma band oscillations, genes that encode Cx36 and Panx2 can be seen as functional candidates for schizophrenia. Moreover, chromosomal locations of both Cx36 (15q14) and Panx2 (22q13) are reported as linkage regions for schizophrenia (Meyer et al. 2002; Kenneth and Lindon 2005). For this reason, the aforementioned genes are positional candidates as well.

\section{Methods}

\section{Sample population}

The sample used in this research consisted of 381 schizophrenia patients (229 males and 151 females; mean age $50 \pm 15.1$ years) and 381 healthy controls (160 males and 221 females; mean age $40 \pm 14.6$ years). For analysis of Cx36, the total sample size consisted of 762 subjects and had been genotyped. On the other hand, the genetic association between schizophrenia and Panx 2 was tested with a subset of the original sample comprised of 384 subjects (case control ratio 1:1). All the subjects were unrelated and of Japanese ethnicity. Subjects included in the case cohort met the DSM-IV criteria for schizophrenia with the consensus of two psychiatrists on the basis of an unstructured diagnostic interview and review of their medical records. Prior to inclusion in the control cohort subjects were screened on the basis of brief diagnostic interviews by an experienced psychiatrist. Subsequent to the study description, written informed consent was requested from each subject. This study was approved by the Ethics Committee at Nagoya University.

\section{SNP selection}

In order to test for genetic association either between the positional or functional candidate and phenotype of interest, we implemented a gene-based approach. This method implies inclusion of both the gene region and gene flanking regions in the association study (Neale and Sham 2004). In other words, it is important to understand the gene as a functional unity of coding and regulatory regions. Later on, by taking advantage of the observed linkage disequilibrium in the region of interest, it was possible to scale down the number of single nucleotide polymorphisms (SNPs) to be included in the association analysis by rejecting the redundant SNPs and picking only haplotype tagging SNPs in accordance with observed linkage disequilibrium (LD).

SNPs were selected from the hapmap database (release \#21; phase II; July 2006, population: Japanese in Tokyo) (HapMap Consortium 2005), and the tagging SNP strategy was based on two criteria: first to exclude redundant SNPs from genotyping by taking advantage of the observed linkage disequlibrium in order to achieve $95 \%$ haplotype coverage and second to exclude SNPs with minor allele frequencies less than $10 \%$ (because of substantial loss of power due to sample size). According to the aforementioned criteria, from initial SNPs, four tagging SNPs for Panx2 (rs17284210, rs3817816, rs4838859 and rs7292533) and two tagging SNPs for Cx36 were selected (rs3743123 and rs752876).

None of the polymorphisms that had been tested in this study were functional; however, we can speculate on the possible impact on gene expression. Moreover, we tried to achieve good coverage of the gene and gene-adjacent regions. The rationale for the latter lies in the fact that tagging SNPs may be used as a proxy for detecting functional polymorphisms (i.e., LD between tagging SNP and unknown functional polymorphisms).

\section{Genotyping methods}

Genomic DNA was extracted from peripheral blood. The restriction fragment length polymorphism (RFLP) method was performed for genotyping rs17284210, rs3817816 and rs7292533. On the other hand, the allelic discrimination assay (Applied Biosystem Japan Ltd., Tokyo) was carried out for genotyping rs4838859, rs3743123 and rs752876. For each 384-well plate used for the allelic discrimination assay, three nontemplate controls were included. Details regarding primer design, cycle condition and restriction enzymes used for RFLP assay, as well as data on the probe design for allelic discrimination assay, are available upon request. 
Statistical methods

The LD blocks were defined in accordance with the four gamete rule (Wang et al. 2002). Haplotype frequencies were estimated by the expectation-maximization algorithm. The aforementioned (LD block definition and haplotype frequency estimation) are functions implemented in the Haploview software v3.32 that was used in this study (Barrett et al. 2005). Genotype deviations from the Hardy-Weinberg equilibrium, allelic and genotypic associations were tested by chi-squared statistics. Log likelihood ratio tests for haplotypic association between schizophrenia and SNPs that are in linkage disequilibrium were carried out by the software Unphased v2.403 (Dudbridge 2003) with a permutation test for the calculation of empirical significance levels for differences between haplotype frequencies in case and control cohorts. The experimental alpha level was set to 0.05 .

Power analysis was performed in accordance with the general power calculation model for chi-squared statistics. In brief, power is determined with respect to the degree of freedom and predefined alpha level of the study, after assuming the effect size (in accordance with Cohen's criteria). For the power calculation, Sample Power software v 2.0 (SPSS inc.) was used.

\section{Results}

HapMap data revealed a discreet linkage disequilibrium pattern in the region of Panx2. In detail, pairwise LD analysis of Panx 2 polymorphisms showed one LD block that comprises several polymorphisms. However, only rs4838859 and rs7292533 were sufficient for 95\% haplotype coverage. In spite of the fact that the remaining two polymorphisms (rs17284210 and rs3817816) are neither in linkage disequilibrium nor within a separate LD block, we included them in the study design in order to achieve satisfactory gene coverage for association analysis. Concerning Cx36, pairwise LD analysis showed one LD block that spreads throughout the gene region. Two SNPs (rs3743123 and rs752876) were sufficient in order to achieve $95 \%$ of haplotype coverage. The LD pattern of our data was in accordance with the LD pattern predicted by the HapMap database data set (data not shown).

Deviation from Hardy-Weinberg equilibrium was not observed. Allele-, genotype- or haplotype-wise analysis did not provide sufficient evidence for association between two genes that encode gap junction proteins and schizophrenia (see Table 1). Regarding Panx2, in case effect size is set to medium (in accordance with Cohen's criteria), the sample that was characterized in our research had approximately $80 \%$ power for detecting genetic association. However, in case the size effect had been set to small, the calculated power was $50 \%$. On the other hand, regarding the Cx36 sample, the power was estimated to be more than $80 \%$ even if the effect size was set to small.

\section{Discussion}

The common disease-common variants hypothesis postulates that linkage disequilibrium should be detected by the

Table 1 Genotype distribution

\begin{tabular}{|c|c|c|c|c|c|c|c|c|c|c|c|}
\hline \multirow[t]{2}{*}{ Gene } & \multirow[t]{2}{*}{$\mathrm{SNP}^{\mathrm{a}}$} & \multirow[t]{2}{*}{ Block $^{b}$} & \multirow[t]{2}{*}{ Cohort } & \multicolumn{3}{|c|}{ Genotype $^{c}$} & \multirow[t]{2}{*}{$P$ value } & \multicolumn{2}{|c|}{ Allele $^{\mathrm{d}}$} & \multirow[t]{2}{*}{$P$ value } & \multirow{2}{*}{$\begin{array}{l}\text { Haplotype } \\
P \text { value }\end{array}$} \\
\hline & & & & $\mathrm{M} / \mathrm{M}$ & $\mathrm{M} / \mathrm{m}$ & $\mathrm{m} / \mathrm{m}$ & & M & $\mathrm{m}$ & & \\
\hline \multirow[t]{4}{*}{ Cx 36} & rs752876 & \multirow[t]{4}{*}{$I_{\mathrm{cx} 36}$} & Case & 121 & 186 & 74 & \multirow[t]{2}{*}{0.807} & 56.2 & 43.8 & \multirow[t]{2}{*}{0.877} & \multirow[t]{4}{*}{0.807} \\
\hline & $\mathrm{G}>\mathrm{A}$ & & Control & 127 & 177 & 77 & & 56.6 & 43.4 & & \\
\hline & rs3743123 & & Case & 138 & 176 & 67 & \multirow[t]{2}{*}{0.803} & 59.3 & 40.7 & \multirow[t]{2}{*}{0.958} & \\
\hline & $\mathrm{C}>\mathrm{T}$ & & Control & 133 & 185 & 63 & & 59.2 & 40.8 & & \\
\hline \multirow[t]{8}{*}{ Panx 2} & rs 17284210 & \multirow[t]{2}{*}{ I } & Case & 134 & 51 & 7 & \multirow[t]{2}{*}{0.680} & 83.1 & 16.9 & \multirow[t]{2}{*}{0.372} & \multirow[t]{2}{*}{ I } \\
\hline & $\mathrm{T}>\mathrm{G}$ & & Control & 141 & 46 & 5 & & 85.4 & 14.6 & & \\
\hline & rs3817816 & \multirow[t]{2}{*}{ I } & Case & 90 & 79 & 23 & \multirow[t]{2}{*}{0.987} & 67.4 & 32.6 & \multirow[t]{2}{*}{0.878} & \multirow[t]{2}{*}{ / } \\
\hline & $\mathrm{G}>\mathrm{A}$ & & Control & 89 & 79 & 24 & & 66.9 & 33.1 & & \\
\hline & rs4838859 & \multirow[t]{4}{*}{$I_{\text {panx } 2}$} & Case & 107 & 74 & 11 & \multirow[t]{2}{*}{0.949} & 75.0 & 25.0 & \multirow[t]{2}{*}{0.802} & \multirow[t]{4}{*}{0.386} \\
\hline & $A>G$ & & Control & 110 & 71 & 11 & & 75.8 & 24.2 & & \\
\hline & rs7292533 & & Case & 84 & 94 & 14 & \multirow[t]{2}{*}{0.344} & 68.2 & 31.8 & \multirow[t]{2}{*}{0.539} & \\
\hline & $\mathrm{T}>\mathrm{C}$ & & Control & 84 & 86 & 22 & & 66.1 & 33.9 & & \\
\hline
\end{tabular}

\footnotetext{
${ }^{\text {a }}$ Upper value is dbSNP designation while lower value represents major $>$ minor allele

${ }^{\mathrm{b}}$ Dotted line represents LD block

c $M$ Major allele, $m$ minor allele in absolute numbers

${ }^{\mathrm{d}} M$ Major allele, $m$ minor allele in percents
} 
haplotype association test if the risk haplotype was linked to variants (Chakravarti 1999). Therefore, regarding the Japanese population, the data presented in this article do not provide sufficient evidence for the involvement of the two gap junction coding genes in conferring that susceptibility.

Definition of phenotypes is vital for genetic association study; therefore, endophenotypes (being more specific than phenotypes) are thought to be important for this field (Gottesman and Gould 2003; Braff et al. 2007). We did not take advantage of endophenotypes in order to test for genetic association, and that might be a limitation of our study. However, here we have to mention that several studies showed gamma band oscillations, recorded in schizophrenic patients, that indeed have reduced power and synchrony in comparison to nonaffected subjects (Spencer et al. 2003, 2004). Therefore, rather that segregation of the sample in respect to the characteristic of gamma band oscillations, we implemented a crude phenotype approach (schizophrenia phenotype in toto) in the study design. Moreover, regarding $\mathrm{Cx} 36$, our data are in concordance with previous research regarding the aforementioned gene and schizophrenia (Meyer et al. 2002).

In conclusion, neither Panx2 nor Cx36 has influence on the pathogenesis of schizophrenia in the Japanese population. However, since this is the first study regarding Panx2 and schizophrenia, it might be interesting to explore the possible association in different population settings.

Acknowledgments We would like to thank Ms Y. Nakamura for her technical support. This work was supported in part by research grants from the Ministry of Education, Culture, Sports, Science and Technology of Japan, the Ministry of Health, Labor and Welfare of Japan, as well as the Japanese Health Sciences Foundation (Research on Health Sciences Focusing on Drug Innovation).

\section{References}

Barrett JC, Fry B, Maller J, Daly MJ (2005) Haploview: analysis and visualization of LD and haplotype maps. Bioinformatics 21:263265

Braff DL, Freedman R, Schork NJ, Gottesman II (2007) Deconstructing schizophrenia: an overview of the use of endophenotypes in order to understand a complex disorder. Schizophr Bull 33:21-32

Bruzzone R, Hormuzdi SG, Barbe MT, Herb A, Monyer H (2003) Pannexins, a family of gap junction proteins expressed in brain. Proc Natl Acad Sci USA 100:13644-13649

Buhl DL, Harris KD, Hormuzdi SG, Monyer H, Buzsaki G (2003) Selective impairment of hippocampal gamma oscillations in connexin-36 knock-out mouse in vivo. J Neurosci 23:1013-1018

Chakravarti A (1999) Population genetics-making sense out of sequence. Nat Genet 21:56-60

Condorelli DF, Belluardo N, Trovato-Salinaro A, Mudo G (2000) Expression of $\mathrm{Cx} 36$ in mammalian neurons. Brain Res Brain Res Rev 32:72-85

Connors BW, Long MA, (2004) Electrical synapses in the mammalian brain. Annu Rev Neurosci 27:393-418
Dudbridge F (2003) Pedigree disequilibrium tests for multilocus haplotypes. Genet Epidemiol. 25:115-121

Gottesman II, Gould TD (2003) The endophenotype concept in psychiatry: etymology and strategic intentions. Am J Psychiatry. 160:636-645

HapMap Consortium (2005) A haplotype map of the human genome. Nature 437:1299-1320

Hong LE, Summerfelt A, McMahon R, Adami H, Francis G, Elliott A, Buchanan RW, Thaker GK (2004) Evoked gamma band synchronization and the liability for schizophrenia. Schizophr Res 70:293-302

Hormuzdi SG, Pais I, LeBeau FE, Towers SK, Rozov A, Buhl EH, Whittington MA, Monyer H, (2001) Impaired electrical signaling disrupts gamma frequency oscillations in connexin 36deficient mice. Neuron 31:487-495

Kenneth KS, Lindon E (2005) Psychiatric genetics. American Psychiatric Publishing, Washington

LeBeau FE, Traub RD, Monyer H, Whittington MA, Buhl EH (2003) The role of electrical signaling via gap junctions in the generation of fast network oscillations. Brain Res Bull 62:3-13

Lewis DA, Hashimoto T, Volk DW (2005) Cortical inhibitory neurons and schizophrenia. Nat Rev Neurosci 6:312-324

Mann EO, Radcliffe CA, Paulsen O (2005) Hippocampal gammafrequency oscillations: from interneurones to pyramidal cells, and back. J Physiol 562:55-63

Meyer J, Mai M, Ortega G, Mossner R, Lesch KP (2002) Mutational analysis of the connexin 36 gene (CX36) and exclusion of the coding sequence as a candidate region for catatonic schizophrenia in a large pedigree. Schizophr Res 58:87-91

Neale BM, Sham PC (2004) The future of association studies: genebased analysis and replication. Am J Hum Genet 75:353-362

Phillips WA, Silverstein SM (2003) Convergence of biological and psychological perspectives on cognitive coordination in schizophrenia. Behav Brain Sci 26:65-82; discussion 82-137

Rozental R, Giaume C, Spray DC (2000) Gap junctions in the nervous system. Brain Res Brain Res Rev 32:11-15

Sadock BJ, Sadock VA (2005) Comprehensive textbook of psychiatry. Lippincott Williams \& Wilkins, Philadelphia

Sohl G, Maxeiner S, Willecke K (2005) Expression and functions of neuronal gap junctions. Nat Rev Neurosci 6:191-200

Spencer KM, Nestor PG, Niznikiewicz MA, Salisbury DF, Shenton ME, McCarley RW (2003) Abnormal neural synchrony in schizophrenia. J Neurosci 23:7407-7411

Spencer KM, Nestor PG, Perlmutter R, Niznikiewicz MA, Klump MC, Frumin M, Shenton ME, McCarley RW (2004) Neural synchrony indexes disordered perception and cognition in schizophrenia. Proc Natl Acad Sci USA. 101:17288-17293

Szabadics J, Lorincz A, Tamas G (2001) Beta and gamma frequency synchronization by dendritic gabaergic synapses and gap junctions in a network of cortical interneurons. J Neurosci 21:58245831

Traub RD, Bibbig A, LeBeau FE, Cunningham MO, Whittington MA (2005) Persistent gamma oscillations in superficial layers of rat auditory neocortex: experiment and model. J Physiol 562:3-8

Waberski TD, Norra C, Kawohl W, Thyerlei D, Hock D, Klostermann F, Curio G, Buchner H, Hoff P, Gobbele R (2004) Electrophysiological evidence for altered early cerebral somatosensory signal processing in schizophrenia. Psychophysiology 41:361366

Wang N, Akey JM, Zhang K, Chakraborty R, Jin L (2002) Distribution of recombination crossovers and the origin of haplotype blocks: the interplay of population history, recombination, and mutation. Am J Hum Genet 71:1227-1234

Whittington MA, Traub RD, Jefferys JG (1995) Synchronized oscillations in interneuron networks driven by metabotropic glutamate receptor activation. Nature 373:612-615 